\title{
High Prevalence of Short-Chain Acyl-CoA Dehydrogenase Deficiency in the Netherlands, but No Association with Epilepsy of Unknown Origin in Childhood
}

\author{
Authors \\ B. T. van Maldegem ${ }^{1}$, S. F. Kloosterman², W. J. Janssen ${ }^{2}$, P. B. Augustijn ${ }^{3}$, J. H. van der Lee ${ }^{4}$, L. IJlst ${ }^{2}$, \\ H. R. Waterham ${ }^{2}$, R Duran ${ }^{2}$, R. J. A. Wanders ${ }^{2}$, F. A. Wijburg \\ Affiliations \\ Affiliation addresses are listed at the end of the article
}

\author{
Key words \\ - short-chain acyl-CoA \\ dehydrogenase deficiency \\ - epilepsy \\ - clinical relevance
}

received $\quad 16.09 .2010$

accepted $\quad$ 09.03.2011

\section{Bibliography}

DOI http://dx.doi.org/

10.1055/s-0031-1275342

Published online:

April 15, 2011

Neuropediatrics 2011;

42: $13-17$

(c) Georg Thieme Verlag KG

Stuttgart - New York

ISSN 0174-304X

Correspondence

\section{B. T. van Maldegem}

Department of Paediatrics Academic Medical Center University of Amsterdam Meibergdreef 9

1105 AZ Amsterdam

The Netherlands

Tel.: + 31/20/566 8890

Fax: + 31/20/691 7735

b.van.maldegem@gelre.nl

\section{Abstract}

$\nabla$

Short-chain acyl-CoA dehydrogenase deficiency (SCADD) is an autosomal recessive inborn error of metabolism, most frequently associated with developmental delay and/or epilepsy. Most SCADD patients carry common SCAD-encoding gene $(A C A D S)$ variants or these variants in combination with a rare ACADS mutation, in the Netherlands predominantly the c. $1058 \mathrm{C}>\mathrm{T}$. Epilepsy in childhood often remains unexplained and patients with epilepsy related to SCADD may remain undiagnosed because studies for SCADD are often not performed. To test this hypothesis and to further estimate the extent of the Dutch SCADD population, we performed a study on

\section{Abbreviations}

$\nabla$

ACADS SCAD-encoding gene

C4-C butyrylcarnitine

C4-CoA butyryl-CoA

EMA ethylmalonic acid

RIVM National Institute for Public Health and the Environment

SCAD short-chain acyl-CoA dehydrogenase

SCADD short-chain acyl-CoA dehydrogenase deficiency

\section{Introduction}

$\nabla$

Short-chain acyl-CoA dehydrogenase (SCAD) is the first enzyme of the short-chain $\beta$-oxidation pathway catalysing dehydrogenation of shortchain fatty acids. SCAD deficiency (SCADD) is a newly recognised autosomal recessive inborn error of fatty acid oxidation that has been associated with a remarkable variety of clinical symptoms. SCADD is probably one of the more common inborn errors of metabolism [23]. blood spot samples in 131 paediatric patients with epilepsy and 909 anonymous newborns and investigated the presence of the 2 common ACADS variants and the rare c. $1058 \mathrm{C}>\mathrm{T}$ mutation. Overall, the 2 common ACADS variants and the rare $\mathrm{c} .1058 \mathrm{C}>\mathrm{T}$ mutation were detected in either homozygous or compound heterozygous forms in $9.2 \%$ of the epilepsy and $7.5 \%$ of the reference group. A birth prevalence of SCADD with a mutation/variant genotype in the Netherlands as high as $>1: 1000$ was calculated. This is in contrast with the low number of patients diagnosed clinically and supports the hypothesis that SCADD is clinically irrelevant. Furthermore our study does not support an association between SCADD and epilepsy.

Butyryl-CoA (C4-CoA) is the main substrate for the SCAD enzyme and accumulates in mitochondria of patients with decreased SCAD activity, which subsequently leads to increased levels of butyrylcarnitine (C4-C) and ethylmalonic acid (EMA). EMA can best be measured in urine, whereas $\mathrm{C} 4 \mathrm{-C}$ can best be measured in plasma and blood spots dried on filter paper. These 2 metabolites constitute the major biochemical features of SCADD.

The diagnosis of SCADD is usually confirmed by DNA analyses [23]. The majority of SCADD patients are homozygotes or compound heterozygotes for 2 common SCAD-encoding gene (ACADS) variants, i.e., c.625G $>$ A and c.511C > T, or for these common ACADS variants in combination with a rare ACADS mutation [20,22-24]. Homozygosity for either of the common ACADS variants has been found in the general population with a remarkably high prevalence: approximately $0.3 \%$ for the common c. $511 \mathrm{C}>\mathrm{T}$ and $5.5 \%$ for the common c.625G $>A$ variant $[17,18]$; and this ACADS genotype has been considered to confer susceptibility to the development of clinical 
disease $[5,9,11]$. More than 70 different ACADS mutations have been reported so far $[5,10,11,14-16,19-21,23,24]$. The majority of Dutch patients are compound heterozygous for the common c.625G > A variant and one specific rare mutation, c. $1058 \mathrm{C}>\mathrm{T}$ [23] located on the c.625G and c.511C ACADS alleles. Overall an $A C A D S$ genotype consisting of this specific rare mutation and/or common ACADS variants was present in $61 \%$ of Dutch SCADD patients [23].

Most of the reported SCADD patients were initially investigated because of neurological symptoms and/or hypoglycaemia $[5,20,22-24]$. In our previous study on 31 Dutch SCADD patients [23], developmental delay was reported in 52\% and epilepsy in $35 \%$ of patients. Behavioural disorders and hypoglycaemia were reported in $26 \%$ and $19 \%$ of this patient group, respectively. No correlation between genotype and phenotype could be established, and in some patients other diagnoses were identified that explained the symptoms initially leading to metabolic studies.

Epilepsy is a frequent finding in childhood. Studies from Europe and North America report rates from 3.6 to 6.5/1000 children $[1-3,6,8,12]$. Diagnostic studies fail to establish the cause of epilepsy in up to $30 \%$ of the patients [13]. However, in patients with idiopathic childhood epilepsy, studies for inborn errors of metabolism such as SCADD, as a potential cause are often not performed. Furthermore, the facts that epilepsy is a frequent symptom in SCADD and that the prevalence of SCADD is much higher than the number of patients detected by metabolic studies [23], suggest that patients with epilepsy related to SCADD may remain undiagnosed.

To test this hypothesis we tested a group of paediatric patients with epilepsy for the presence of SCADD. We aimed to gain knowledge regarding the need for SCADD studies in epilepsy patients. In addition, we aimed to further estimate the extent of SCADD individuals in the Netherlands by establishing the frequencies of the common c.511C $>$ T ACADS variant and the rare c. $1058 \mathrm{C}>\mathrm{T}$ ACADS mutation in the Dutch population.

\section{Patients and Methods \\ V}

\section{Patients and material}

We performed a study involving 131 paediatric patients with epilepsy and 909 anonymous newborns. Blood spots for the reference group were obtained from the RIVM (National Institute for Public Health and the Environment, the Dutch Institute involved in neonatal screening). The sample of newborn screening cards used was proportional to the number of live births in each of 14 screening districts, ensuring adequate demographic representation of the Dutch population. Dutch newborn screening cards are not released for any reason except newborn screening testing during the first 5 years. Consequently, the screening cards used in this study were more than 5 years old. Newborn screening cards were stored at $4^{\circ} \mathrm{C}$ and they were analysed anonymously.

The study was performed in a paediatric patient group with patients aged 16 years or younger at the time of participation. Because all patients in the Dutch SCADD patients group were $<8$ years old when epilepsy symptoms started, we restricted the epilepsy group to those with onset of epilepsy at $<8$ years. The exclusion criteria included a known or suspected cause of the epilepsy and any previously performed metabolic studies (including SCADD investigations).
Patients with epilepsy were recruited via paediatricians and neurologists within the Academic Medical Center Amsterdam, and 3 Dutch epilepsy centres. Patient and symptom characteristics were obtained using questionnaires answered by parents or legal representatives.

Written informed consent was obtained from all patients and/or parents/legal representatives of the patients. The study protocol was approved by the Medical Ethics Committee of the Academic Medical Center in Amsterdam and the RIVM.

\section{DNA PCR-RFLP analysis}

DNA was extracted from blood spot samples using Chelex 100 (BioRad) essentially as previously described [7,25], but with the following modifications. Blood spot samples ( $6 \mathrm{~mm}$ diameter) were washed overnight at $4^{\circ} \mathrm{C}$ with $1 \mathrm{~mL}$ sterile water. The next day, the supernatant was discarded, $200 \mu \mathrm{L}$ of Chelex $(50 \mathrm{~g} / \mathrm{L}, \mathrm{pH}$ 10.6) were added and the sample was incubated at $56^{\circ} \mathrm{C}$ for $30 \mathrm{~min}$. Subsequently, the samples were mixed for $10 \mathrm{~s}$, centrifuged ( $3 \mathrm{~min}$ at $10000 \times \mathrm{g}$ ), incubated for $8 \mathrm{~min}$ at $96^{\circ} \mathrm{C}$, mixed again for $10 \mathrm{~s}$ and centrifuged ( $3 \mathrm{~min}$ at $10000 \times \mathrm{g}$ ). From this mixture, $14.5 \mu \mathrm{L}$ of supernatant were used in a $25-\mu \mathrm{L}$ PCR reaction.

The extracted DNA was subjected to PCR-RFLP analysis to determine the presence of the common c.625G $>\mathrm{A}$ and c.511C $>\mathrm{T}$ ACADS variants and the rare $\mathrm{c} .1058 \mathrm{C}>\mathrm{T}$ ACADS mutation. Concerning the common c.625G $>$ A variant, a RFLP assay was used as previously described [17]. To identify the common c.511C > T variant and the rare c. $1058 \mathrm{C}>\mathrm{T}$ mutation, new PCR-RFLP assays were developed.

The common c.511C > T variant creates an MvaI restriction site. Exon 5 contains an additional Mval site $42 \mathrm{bp}$ downstream that is used as an internal control for restriction. The upstream endogenous $\mathrm{Mval}$ site was destroyed by a C>A change (bold) in the forward primer in order to increase the size of the restriction product. For the PCR-RFLP analysis, exon 5 of the ACADS gene is amplified from $14.5 \mu \mathrm{L}$ extracted DNA in a $25 \mu \mathrm{L}$ PCR reaction as previously described [7], except for the use of $1.25 \mathrm{mM} \mathrm{MgCl}_{2}$. The following primers were used: forward 5'-tgtaaaacgacggccagt CGT GCG CTG AGC ACT GGG TCT-3', reverse 5V-caggaaacagctatgacc TCG AAG CCT CCC AGG CAT TGG TGA-3'. Similar to the reverse primer for the PCR-RFLP analysis of the common c.625G $>$ A variant, the forward and reverse primers for determining the common c.511C $>\mathrm{T}$ variant have an M13-rev and -21M13 extension (lower case letters), that can be used for sequence analysis.

The rare c.1058C > T mutation creates an Eco130I (StyI) restriction site. Exon 9 contains an additional Eco130I (StyI) site $18 \mathrm{bp}$ upstream that is used as an internal control for restriction. For this PCR-RFLP analysis, exon 9 of the ACADS gene is amplified from $14.5 \mu \mathrm{L}$ extracted DNA in a $25 \mu \mathrm{L}$ PCR reaction as previously described [17] using $1.0 \mathrm{mM} \mathrm{MgCl}_{2}$ and using 5'-tgtaaaacgacggccagt GGG AAG GCT CTG ACT GTA CC-3' as forward primer and 5'-caggaaacagctatgacc CAG GAT CTG GAT GGC CTG AG-3' as reverse primer. These primers also have an M13-rev and -21M13 extension (lower case letters).

For DNA amplification a PTC 100 thermocycler was programmed as follows: denaturation for $2 \mathrm{~min}$ at $96^{\circ} \mathrm{C}$, followed by 5 cycles of $30 \mathrm{~s}$ at $96^{\circ} \mathrm{C}, 30 \mathrm{~s}$ at $55^{\circ} \mathrm{C}$ and $30 \mathrm{~s}$ at $72^{\circ} \mathrm{C}$; this is followed by 30 cycles of $30 \mathrm{~s}$ at $94^{\circ} \mathrm{C}, 30 \mathrm{~s}$ at $55^{\circ} \mathrm{C}$ and $30 \mathrm{~s}$ at $72^{\circ} \mathrm{C}$ with a final step of $15 \mathrm{~min}$ at $72^{\circ} \mathrm{C}$. 
Table 1 Frequency of ACADS genotypes in study populations and in the epilepsy group.

\begin{tabular}{|c|c|c|c|c|c|}
\hline & \multirow{2}{*}{$\begin{array}{l}\text { Nagan study [18] } \\
\qquad(n=694)\end{array}$} & \multirow{2}{*}{$\begin{array}{l}\text { Previous study [17] } \\
\qquad(n=1036)\end{array}$} & \multicolumn{2}{|c|}{ Current study $(n=909)$} & \multirow{2}{*}{$\begin{array}{c}\text { Epilepsy group } \\
\qquad(\mathrm{n}=131)\end{array}$} \\
\hline & & & Observed & Expected* & \\
\hline $511 \mathrm{C} / \mathrm{C}$ & & & $91.0 \%$ & $91.1 \%$ & $90.8 \%$ \\
\hline $511 \mathrm{~T} / \mathrm{C}$ & $5.6 \%$ & & $8.9 \%$ & $8.7 \%$ & $9.2 \%$ \\
\hline $511 \mathrm{~T} / \mathrm{T}$ & $0.3 \%$ & & $0.1 \%$ & $0.2 \%$ & $0 \%$ \\
\hline $625 G / G$ & & & $59.7 \%$ & $59.7 \%$ & $55.7 \%$ \\
\hline $625 \mathrm{~A} / \mathrm{G}$ & $31.8 \%$ & $31.3 \%$ & $35 \%$ & $35 \%$ & $36.6 \%$ \\
\hline $625 \mathrm{~A} / \mathrm{A}$ & $5.6 \%$ & $5.5 \%$ & $5.3 \%$ & $5.3 \%$ & $7.6 \%$ \\
\hline $1058 \mathrm{C} / \mathrm{C}$ & & & $99.3 \%$ & $99.4 \%$ & $99.2 \%$ \\
\hline $1058 \mathrm{~T} / \mathrm{C}$ & & & $0.7 \%$ & $0.6 \%$ & $0.8 \%$ \\
\hline \multicolumn{6}{|l|}{ combinations } \\
\hline $1058 \mathrm{~T} / \mathrm{C}+625 \mathrm{~A} / \mathrm{G}$ & & & $0.1 \%$ & $0.07 \%$ & $0 \%$ \\
\hline $625 \mathrm{~A} / \mathrm{G}+511 \mathrm{~T} / \mathrm{C}$ & $1.0 \%$ & & $2 \%$ & $1.04 \%$ & $1.5 \%$ \\
\hline $511 \mathrm{~T} / \mathrm{T}, 625 \mathrm{~A} / \mathrm{A}, 1058 \mathrm{~T} / \mathrm{C}+625 \mathrm{~A} / \mathrm{G}, 625 \mathrm{~A} / \mathrm{G}+511 \mathrm{~T} / \mathrm{C}$ & & & $7.5 \% * *$ & $6.6 \%$ & $9.2 \% * *$ \\
\hline
\end{tabular}

RFLP c.625G > A: To $25 \mu \mathrm{L}$ of PCR product, $2.9 \mu \mathrm{L}$ Buffer M and $5 \mathrm{U}$ DdeI (Roche) were added, and these additions were followed by overnight incubation at $37^{\circ} \mathrm{C}$. In the presence of the c.625G variant, 2 restriction fragments ( 208 and $18 \mathrm{bp}$ ) are observed; in the presence of the c.625A variant, 3 fragments $(180,28$, and $18 \mathrm{bp}$ ) are observed. Thus, heterozygosity for the common c. $625 \mathrm{G}>$ A variant will yield fragments of $208,180,28$, and $18 \mathrm{bp}$. The undigested PCR fragment is $226 \mathrm{bp}$.

RFLP C.511C>T: To $25 \mu \mathrm{L}$ of PCR product $2.9 \mu \mathrm{L}$ Buffer R and 10U Mval (Fermentas) were added, and these additions were followed by overnight incubation at $37^{\circ} \mathrm{C}$. The undigested PCR fragment of the c. $511 \mathrm{C}>\mathrm{T}$ variant is $175 \mathrm{bp}$. The digested PCR product shows 2 fragments ( 130 and $45 \mathrm{bp}$ ) in a non-carrier of the common c.511C > T variant and fragments of $130,88,45$, and 42 bp when the common c. $511 \mathrm{C}>\mathrm{T}$ variant is present in one of the alleles.

RFLP $1058 \mathrm{C}>\mathrm{T}$ : To $25 \mu \mathrm{L}$ of PCR product $2.9 \mu \mathrm{L}$ Buffer $\mathrm{O}$ and $10 \mathrm{U}$ Eco130I (StyI) (Fermentas) were added, and these conditions were followed by overnight incubation at $37^{\circ} \mathrm{C}$. The undigested PCR fragment of the rare c. $1058 \mathrm{C}>\mathrm{T}$. mutation is $238 \mathrm{bp}$. The digested PCR product shows 2 fragments ( 179 and $59 \mathrm{bp}$ ) in the wild-type and and 4 fragments (179, 161, 59, and 18bp) when the c.1058C > T mutation is present in heterozygous form. Restriction fragments were analysed on a $2 \%(\mathrm{w} / \mathrm{v})$ agarose gel stained using ethidium bromide. Before loading 1/10 volume loading dye $(150 \mathrm{~g} / \mathrm{L}$ Ficoll $400,1.5 \mathrm{~g} / \mathrm{L}$ orange $\mathrm{G}$, and $0.14 \mathrm{~g} / \mathrm{L}$ xylene cyanol FF) was added to samples. A 100 bp ladder (Invitrogen) was used to estimate fragment sizes.

\section{Statistical analyses}

SPSS (SPSS 12.0.1), Graphpad Prism 3.0 and CIA (version 1, 1989) software programmes were used to analyse the data from the DNA studies.

The sample size necessary for this study was calculated based on previous studies that estimate that $6 \%$ of normal (reference) individuals have ACADS genotypes that are either homozygous or compound heterozygous for the common ACADS variants and/or the rare c.1058C $>$ T mutation $[17,18,23]$. A 3-fold higher frequency, i.e., $18 \%$ in the patient group was supposed to indicate an association between these specific ACADS genotypes and epilepsy. Using a 2-tailed test with $\alpha=0.05$ and power $=80 \%$, we estimated that a sample size of at least 65 patients and 260 reference subjects was required.

To estimate the association between the ACADS genotypes and epilepsy, the odds ratio was calculated.

\section{Results \\ $\nabla$}

Incidence of the common c.511C > T and c.625G > A ACADS gene variants and the rare $c .1058 C>T$ mutation in the Dutch population reference group

The distribution of genotypes determined for the common c. $511 \mathrm{C}>\mathrm{T}$ variant clearly favoured the wild-type allele. In 81 of the 909 individuals $(8.9 \%)$ one copy of the common c.511C > T variant was identified and one individual $(0.1 \%)$ was homozygous for this variant ( Table 1). Homozygosity and heterozygosity for the common c.625G > A variant were far more prevalent, with percentages of 5.3 and 35\%, respectively. In 18 of the 909 individuals ( $2 \%$ ) compound heterozygosity for the 2 different common variants was established. In 6 of the 909 individuals $(0.7 \%)$ one copy of the rare c. $1058 \mathrm{C}>\mathrm{T}$ mutation was identified. One of these 6 individuals also carried one copy of the common c.625G $>$ A gene variant. Based on the observed allele frequencies of $4.6,22.8$ and $0.3 \%$ for the common c. $511 \mathrm{C}>\mathrm{T}$ and c.625G $>\mathrm{A}$ gene variants and the rare c. $1058 \mathrm{C}>\mathrm{T}$ mutation, respectively, the expected genotype frequencies were calculated, assuming Hardy-Weinberg equilibrium ( $\bullet$ Table 1).

Overall, the common c.511C $>$ T or c.625G $>$ A gene variant or the rare $c .1058 \mathrm{C}>\mathrm{T}$ mutation were detected in either homozygous or compound heterozygous forms along with 1 of the other 2 in $68 / 909$ (7.5\%, 95\% CI 5.9-9.4\%) subjects from the Dutch population.

\section{Demographic data of the epilepsy study group}

Data from 131 epilepsy patients (66 girls and 85 boys) were studied. Age at study inclusion ranged between 0 and 16 years (median: 9 years). Age of first epileptic insult ranged from 0 to 6 years (median: 3 years). In all patients the epilepsy was defined as idiopathic. Normal development was confirmed in 92/131 (70\%) patients and 39/131 (30\%) patients had delayed cognitive and/or motor function. 
Incidence of the common c.511C > T and c.625G > A ACADS gene variants and the c. $1058 \mathrm{C}>\mathrm{T}$ mutation in the epilepsy study group and comparison with the reference group

In 12 of the 131 patients (9.8\%), one copy of the common c.511C $>\mathrm{T}$ variant was identified, but none of the epilepsy patients were homozygous for this variant ( $\bullet$ Table 1 ). The common c.625G > A variant was identified in heterozygous form in 48 patients (36.1\%) and in homozygous form in $10(7.5 \%)$ patients. One of the patients ( $0.8 \%$ ) carried one copy of the rare c.1058C $>$ T mutation. Overall, the common c.511C $>$ T variant, the common c.625G > A variant or the rare c.1058C $>$ T mutation were detected in either homozygous or compound heterozygous forms along with 1 of the other 2 in 12/131 (9.2\%, 95\% CI 4.8$15.5 \%)$ epilepsy patients. This was comparable to the reference group (7.5\%, ○ Table 1). Within the epilepsy group, the combined ACADS genotypes were established in $4 / 39$ (10\%, 95\% CI 2.9-24.2\%) patients with developmental delay and in 8/92 (8.7\%, $95 \%$ CI $3.8-16.4 \%$ ) patients without developmental delay.

\section{Discussion}

$\nabla$

The current study is the first to investigate the prevalence of the common c.511C > T ACADS variant and the rare c.1058C > T ACADS mutation in the Netherlands. In addition, it is the first study to examine a potential association between SCADD and one of its most frequently associated symptoms: epilepsy. This study included 131 patients and 909 reference subjects, resulting in a power of $>98 \%$ to detect a difference of at least $12 \%$ between the reference and the patient groups.

Homozygosity and heterozygosity for the common c.511C > T ACADS variant in the Dutch population $(0.1$ and $8.9 \%$, respectively) were comparable to the percentages reported for the USA population ( $\triangle$ Table 1) [18]. The prevalence of the most common ACADS variant, c.625G $>\mathrm{A}$, in the Netherlands, has been investigated previously [17], and the current study provides similar results (5.3 and 35\%, respectively, 0 Table 1). Because those results are also comparable to numbers reported for the USA population, it appears that both common ACADS variants, which have been considered to confer susceptibility to the development of clinical SCADD, are represented proportionally throughout the Western world. If these common ACADS variants would indeed confer susceptibility for clinical SCADD, this would imply every 1:14 Western newborns to be at risk for "SCADD symptoms". In the Netherlands, which has an annual birth rate of 180000 [4], this corresponds to approximately 12500 newborns each year.

In addition we investigated the prevalence of the rare c.1058C $>\mathrm{T}$ mutation, which is thought to represent a Dutch founder mutation [23]. The results of the current study provide evidence for a founder mutation because $0.7 \%$ percent of the reference group was identified with one c.1058C > T copy, all patients carrying the rare c.1058C $>$ T mutation were of Dutch ancestry, and as this rare mutation has not been reported in patients without Dutch ancestors [23]. Based on the established frequencies for this rare Dutch mutation and the common ACADS variants, a birth prevalence as high as 1:1000 for an ACADS genotype compound heterozygous for an common ACADS variant plus the rare c.1058C $>T$ mutation, can be calculated. This ACADS genotype has been associated with the biochemical features (increased C4-C and/or EMA) of SCADD in all previously reported cases [23]. For the
Netherlands this implies that, in addition to approximately 12500 newborns with supposed susceptibility for clinical SCADD, 180 newborns with SCADD would be born every year. The results of our study demonstrate that the combined prevalence of all SCADD-related genotypes in the Netherlands amounts to at least 1:1000, which is significantly higher than our previous estimate of 1:3300 [23]. This is in strong contrast with the fewer than 40 patients with SCADD who have been diagnosed in the Netherlands in recent decades. Failure to diagnose SCADD may be explained if SCADD often presents with clinical signs and symptoms that do not lead to metabolic studies of urine or plasma for increased EMA and/or C4-C concentrations. Because epilepsy appears to be a common clinical symptom in SCADD, and uncomplicated epilepsy in childhood is usually not followed by diagnostic tests for metabolic diseases, a high prevalence of SCADD in patients with unexplained epilepsy may, in part, explain the high number of unrecognised SCADD patients. However, failure to diagnose SCADD may also be explained if SCADD only represents a biochemical condition, not leading to any symptoms.

We therefore investigated the presence of the most common ACADS genotypes (representing 61\% percent of ACADS genotypes in the Dutch SCADD patient group) in paediatric patients with epilepsy of unknown origin as well as in reference subjects. However, our study failed to demonstrate an association between SCADD, defined as homozygosity or compound heterozygosity for the common c.511C>T and c.625G >A variant and/or the Dutch rare c.1058C > T mutation and epilepsy of unknown origin in childhood.

A first limitation of our study is that we could not exclude an association between idiopathic childhood epilepsy and SCADD with rare mutations on both alleles. In order to do this very high numbers of patients and reference subjects would have been needed, due to the fact that homozygosity or compound heterozygosity for these mutations is rare. A second limitation is that we cannot rule out an association of epilepsy with the studied ACADS genotypes with an OR smaller than 2.4. However, given the results of our study the probability of an OR larger than 2.4 is only $2.5 \%$ and therefore not very likely.

In conclusion, our study shows that the prevalence of SCADD in the Netherlands is at least $1 / 1000$ newborns. Because this is in contrast with the low number of patients diagnosed clinically, it contributes to the hypothesis that SCADD has no clinical relevance. Our study does not provide evidence for an association between SCADD based on the most common ACADS genotypes and epilepsy. This suggests that SCADD is not an important risk factor for the development of epilepsy and metabolic investigations aimed to diagnose SCADD for childhood epilepsy of unknown origin cannot be recommended.

\section{Acknowledgements \\ $\nabla$}

The authors thank the epilepsy patients and their parents for their participation in this study; the RIVM for providing the neonatal screening cards; Dr. H. Geesink, Prof. Dr. B.T. Poll-The and Dr. W. C. G. Plandsoen for participating in this study; and Hetty Wijnia, Anette van Steenbergen and Mieke Stolwijk from the Stichting Epilepsie Instellingen Nederland and Thessa Westphal and Klaske Honig from the Department of Paediatrics/Metabolic Diseases of the AMC for their administrative support. This work was supported by Sigma-Tau B.V., "Metakids" (www.metakids. 
$\mathrm{nl}$, the Dutch association for research on metabolic disorders), "Stichting Emma Kinderziekenhuis", and "Stichting A.C. Thomsenfonds".

\section{Affiliations}

${ }^{1}$ Department of Paediatrics, Academic Medical Center, University of Amsterdam, Amsterdam, The Netherlands

${ }^{2}$ Laboratory Genetic Metabolic Diseases, Academic Medical Center, University of Amsterdam, Amsterdam, The Netherlands

${ }^{3}$ Meer en Bosch and de Cruquishoeve, Stichting Epilepsie Instellingen Nederland, Heemstede, The Netherlands

${ }^{4}$ Department of Paediatric Clinical Epidemiology, Academic Medical Center, University of Amsterdam, Amsterdam, The Netherlands

\section{References}

1 Baumann RJ, Marx MB, Leonidakis MG. Epilepsy in rural Kentucky: prevalence in a population of school age children. Epilepsia 1978; 19: $75-80$

2 Beilmann A, Napa A, Soot A et al. Prevalence of childhood epilepsy in Estonia. Epilepsia 1999; 40: 1011-1019

3 Cavazzuti GB. Epidemiology of different types of epilepsy in school age children of Modena, Italy. Epilepsia 1980; 21: 57-62

4 Centraal Bureau voor de Statistiek. http://statline.cbs.nl/StatWeb/ publication $/$ ?VW $=\mathrm{T} \& \mathrm{DM}=\mathrm{SLNL} \& \mathrm{PA}=37422$ ned $\& \mathrm{D} 1=0,4-5,7,9,11$, $13,17,26,35,40-41 \& D 2=0,10,20,30,40,(1-4)-1 \& H D=090218-0953 \& H D R=$ $\mathrm{G} 1 \& \mathrm{STB}=\mathrm{T} .2010$

5 Corydon MJ, Vockley J, Rinaldo P et al. Role of common gene variations in the molecular pathogenesis of short-chain acyl-CoA dehydrogenase deficiency. Pediatr Res 2001; 49: 18-23

6 Cowan LD. The epidemiology of the epilepsies in children. Ment Retard Dev Disabil Res Rev 2002; 8: 171-181

7 den Boer ME, Ijlst L, Wijburg FA et al. Heterozygosity for the common LCHAD mutation $(1528 \mathrm{~g}>\mathrm{C})$ is not a major cause of HELLP syndrome and the prevalence of the mutation in the Dutch population is low. Pediatr Res 2000; 48: 151-154

8 Eriksson KJ, Koivikko MJ. Prevalence, classification, and severity of epilepsy and epileptic syndromes in children. Epilepsia 1997; 38: 1275-1282

9 Gregersen N, Andresen BS, Bross P. Prevalent mutations in fatty acid oxidation disorders: diagnostic considerations. Eur J Pediatr 2000; 159 (Suppl 3): S213-S218

10 Gregersen $N$, Andresen BS, Pedersen CB et al. Mitochondrial fatty acid oxidation defects - remaining challenges. J Inherit Metab Dis 2008: 31: 643-657

11 Gregersen N, Winter VS, Corydon MJ et al. Identification of four new mutations in the short-chain acyl-CoA dehydrogenase (SCAD) gene in two patients: one of the variant alleles, $511 \mathrm{C}->\mathrm{T}$, is present at an unexpectedly high frequency in the general population, as was the case for $625 \mathrm{G}->\mathrm{A}$, together conferring susceptibility to ethylmalonic aciduria. Hum Mol Genet 1998; 7: 619-627
12 Hauser WA, Annegers JF, Kurland LT. Prevalence of epilepsy in Rochester, Minnesota: 1940-1980. Epilepsia 1991; 32: 429-445

13 Jallon $P$, Latour P. Epidemiology of idiopathic generalized epilepsies. Epilepsia 2005; 46 (Suppl 9): 10-14

14 Jethva R, Bennett MJ, Vockley J. Short-chain acyl-coenzyme A dehydrogenase deficiency. Mol Genet Metab 2008; 95: 195-200

15 Koeberl DD, Young SP, Gregersen NS et al. Rare disorders of metabolism with elevated butyryl- and isobutyryl-carnitine detected by tandem mass spectrometry newborn screening. Pediatr Res 2003; 54: 219-223

16 Kristensen MJ, Kmoch S, Bross $P$ et al. Amino acid polymorphism (Gly209Ser) in the ACADS gene. Hum Mol Genet 1994; 3: 1711

17 Maldegem BT, Waterham HR, Duran M et al. The 625G >A SCAD gene variant is common but not associated with increased C4-carnitine in newborn blood spots. J Inherit Metab Dis 2005; 28: 557-562

18 Nagan N, Kruckeberg KE, Tauscher AL et al. The frequency of shortchain acyl-CoA dehydrogenase gene variants in the US population and correlation with the $C(4)$-acylcarnitine concentration in newborn blood spots. Mol Genet Metab 2003; 78: 239-246

19 Naito E, Indo $Y$, Tanaka K. Identification of two variant short chain acyl-coenzyme A dehydrogenase alleles, each containing a different point mutation in a patient with short chain acyl-coenzyme A dehydrogenase deficiency. J Clin Invest 1990; 85: 1575-1582

20 Pedersen $C B$, Kolvraa S, Kolvraa $A$ et al. The ACADS gene variation spectrum in 114 patients with short-chain acyl-CoA dehydrogenase (SCAD) deficiency is dominated by missense variations leading to protein misfolding at the cellular level. Hum Genet 2008; 124: 43-56

21 Seidel J, Streck S, Bellstedt $K$ et al. Recurrent vomiting and ethylmalonic aciduria associated with rare mutations of the short-chain acyl-CoA dehydrogenase gene. J Inherit Metab Dis 2003; 26: 37-42

22 Tein I, Elpeleg O, Ben-Zeev B et al. Short-chain acyl-CoA dehydrogenase gene mutation $(\mathrm{c} .319 \mathrm{C}>\mathrm{T})$ presents with clinical heterogeneity and is candidate founder mutation in individuals of Ashkenazi Jewish origin. Mol Genet Metab 2008; 93: 179-189

23 van Maldegem BT, Duran M, Wanders RJ et al. Clinical, biochemical, and genetic heterogeneity in short-chain acyl-coenzyme A dehydrogenase deficiency. JAMA 2006; 296: 943-952

24 Waisbren SE, Levy HL, Noble M et al. Short-chain acyl-CoA dehydrogenase (SCAD) deficiency: an examination of the medical and neurodevelopmental characteristics of 14 cases identified through newborn screening or clinical symptoms. Mol Genet Metab 2008; 95: 39-45

25 Walsh PS, Metzger DA, Higuchi R. Chelex 100 as a medium for simple extraction of DNA for PCR-based typing from forensic material. Biotechniques 1991; 10: 506-513 